\title{
Degeneração macular relacionada à idade: prevalência e fatores de risco em dois centros oftalmológicos de referência em Pernambuco
}

\author{
Age-related maculardegeneration:analysis in twoophthalmologicalcenters in \\ Pernambuco-Brazil
}

\author{
Laura Patrícia Ferreira Santos ${ }^{1}$ \\ José Ricardo Diniz ${ }^{2}$ \\ Ana Cecília de Souza Leão ${ }^{3}$ \\ Marília Fernandes de Sena ${ }^{4}$
}

\footnotetext{
${ }^{1}$ Residente em Oftalmologia na Fundação Altino Ventura -FAV. Recife (PE).

${ }^{2}$ Médico oftalmologista do Hospital de Olhos de Pernambuco. Recife (PE).

Residente em Oftalmologia na FAV. Recife (PE).

${ }^{4}$ Residente em Oftalmologia na FAV. Recife (PE)

Endereço para correspondência: Rua Samuel de Farias,

122 - Apt 201B - Recife (PE) CEP 52060-430

E-mail: laurasabino@ig.com.br

Recebido para publicação em 02.04.2004

Versão revisada recebida em 22.10.2004

Aprovação em 03.01.2005
}

\begin{tabular}{|c|}
\hline RESUMO \\
\hline 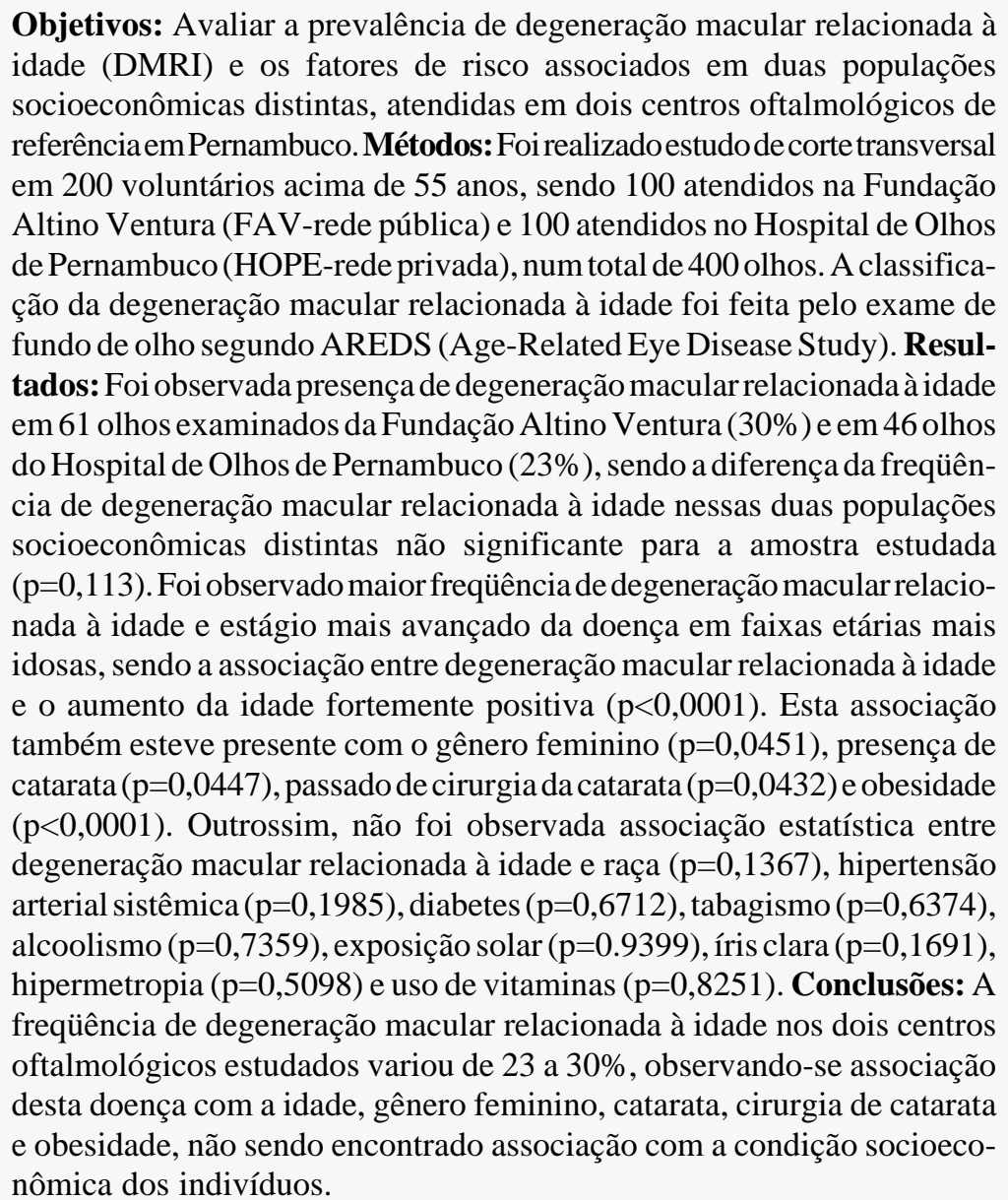 \\
\hline
\end{tabular}

Descritores: Degeneração macular/epidemiologia; Envelhecimento; Idoso; Fatores de risco; Obesidade; Alcoolismo; Tabagismo; Fatores socioeconômicos

\section{INTRODUÇÃO}

A degeneração macular relacionada à idade (DMRI) é a principal causa de cegueira na população acima de 55 anos. Sua prevalência aumenta com a idade afetando cerca de 8,5 a 27,9\% da população maior que $75 \operatorname{anos}^{(1)}$. Tem 
sido observado que a incidência dessa doença vem aumentando nas últimas décadas na ordem de 30 a $40 \%$, apesar de doenças oftalmológicas como a catarata e glaucoma, que atingem a mesma faixa populacional, terem apresentado, aparentemente, redução dos seus registros ${ }^{(2)}$.

Apesar da etiologia não ter sido ainda determinada, tem sido mostrado que fatores ambientais e genéticos podem estar envolvidos ${ }^{(1)}$. Um insulto oxidativo que resulta na morte de fotorreceptores da mácula poderia ser a gênese do processo(3) Observa-se, em vários estudos populacionais, que fatores de risco estão relacionados a DMRI. Idade ${ }^{(4)}$ e exposição ao fumo ${ }^{(4-5)}$ são fatores constantes de estudos recentes. Outros fatores relatados envolvem hipertensão arterial, uso de inibidores de enzima conversora de angiotensina, hipermetropia, catarata e cirurgia de catarata ${ }^{(4,6)}$, obesidade ${ }^{(7)}$, cor clara de íris, exposição ocular à luz e consumo de álcool ${ }^{(4,8)}$. Tem sido observado, em estudos populacionais, que medidas preventivas como mudanças dietéticas podem reduzir o risco individual de desenvolvimento de DMRI ${ }^{(9)}$. A terapia de reposição de zinco e antioxidantes (vitamina $\mathrm{C}$, E, e beta-carotenos) tem sido efetiva na prevenção da perda visual severa por DMRI em pacientes selecionados ${ }^{(10)}$.

Não existe terapia efetiva para a forma atrófica da DMRI, que acomete mais de $90 \%$ dos pacientes com essa condição ${ }^{(11)}$. Para os $10 \%$ restantes, a terapia com fotocoagulação a laser e a terapia fotodinâmica com verteporfina permitem relativa estabilização da visão em cerca de $2 / 3$ dos olhos, apesar dos altos $\operatorname{custos}^{(1)}$. Portanto, a prevenção é a melhor estratégia, uma vez que a DMRI reduz a habilidade individual de realizar as atividades diárias que requeiram visão central nítida, estando associada com elevado risco de depressão e dependência social ${ }^{(12)}$, o que vem representar um importante impacto socioeconômico para o estado ${ }^{(13)}$.

Constituiu objetivo da presente análise avaliar a prevalência de DMRI e os fatores de risco associados em duas populações socioeconômicas distintas, atendidas em dois centros oftalmológicos de referência em Pernambuco.

\section{MÉTODOS}

Foi realizado um estudo de corte transversal em 200 voluntários acima de 55 anos, sendo 100 atendidos pela Fundação Altino Ventura (rede pública) e 100 atendidos pelo Hospital de Olhos de Pernambuco (rede privada), no período de fevereiro de 2003 a outubro de 2003, perfazendo um total de 400 olhos examinados. Esses indivíduos foram incluídos no estudo após autorização e assinatura do termo de consentimento esclarecido.

Foram avaliados nos antecedentes pessoais a presença de diabetes e hipertensão arterial sistêmica. História positiva para tabagismo e alcoolismo foi considerada se ocorreram em um período superior a 1 ano. Em relação ao índice de massa corpórea (IMC), obtido quando dividido o peso sobre a altura ao quadrado, foi considerado IMC magro quando menor que
20; normal quando entre 20 e 24; acima do peso quando entre 25 e 29 e obeso quando maior que 29 . Em relação ao uso de vitaminas foi considerada positiva quando havia ingestão regular, de qualquer tipo de vitamina, por período superior a 1 ano. Em relação à exposição solar, foi considerada positiva quando os pacientes apresentavam antecedentes de exposição ocupacional ao sol, ou exposição solar regular por período superior a cinco anos.

O exame oftalmológico compreendeu a avaliação da acuidade visual de longe, medida de forma monocular e com melhor correção óptica, utilizando a tabela de Snellen a 6 metros, biomicroscopia, tonometria de aplanação (Goldman), fundoscopia sob midríase com lente de 78DE, com atenção especial à região macular, e exame com tela de Amsler.

A classificação da DMRI foi feita separadamente em cada olho, segundo critérios do AREDS ${ }^{10}$. Para efeito de apresentação de resultados e análise de dados, o estágio 1 da doença foi denominado sem DMRI, estágio 2 (DMRI inicial), estágio 3 e 4 (DMRI estabelecida).

As variáveis qualitativas foram expressas por suas freqüências. Possíveis diferenças entre freqüências foram avaliadas pelo teste do qui-quadrado e teste exato de Fisher. As variáveis quantitativas foram expressas por suas médias e desvios padrões (DP). Foi utilizado $\mathrm{p}<0,05$ para rejeição da hipótese de nulidade ${ }^{(14)}$.

\section{RESULTADOS}

Foi observada presença de DMRI em 61 olhos examinados da FAV (30\%) e em 46 olhos do HOPE (23\%), sendo a diferença da freqüência de DMRI nessas duas populações socioeconômicas distintas não significante para a amostra estudada $(\mathrm{p}=0,113)$. A frequiência de DMRI e sua distribuição, segundo instituição analisada, estão expressas na tabela 1.

Em relação à idade da população estudada foi observada maior frequiência de olhos com DMRI e estágio mais avançado da doença em faixas etárias mais idosas em ambas instituições, sendo a associação entre DMRI e o aumento da idade fortemente positiva $(\mathrm{p}<0,0001)$. A distribuição das frequiências da doença nas populações estudadas, segundo faixa etária, está expressa na tabela 2 .

\begin{tabular}{|c|c|c|c|c|}
\hline \multicolumn{5}{|c|}{$\begin{array}{l}\text { Tabela 1. Freqüência de olhos com degeneração macular relacionada } \\
\text { à idade (DMRI) e estágio da doença na Fundação Altino Ventura (FAV) } \\
\text { e Hospital de Olhos de Pernambuco (HOPE) }\end{array}$} \\
\hline & \multicolumn{2}{|c|}{ FAV } & \multicolumn{2}{|c|}{ HOPE } \\
\hline & $\mathbf{n}$ & $\%$ & $\mathbf{n}$ & $\%$ \\
\hline Sem DMRI & 139 & 70 & 154 & 77 \\
\hline \multicolumn{5}{|l|}{ Com DMRI } \\
\hline Inicial & 26 & 13 & 16 & 8 \\
\hline Estabelecida & 35 & 17 & 30 & 15 \\
\hline Total & 200 & 100 & 200 & 100 \\
\hline
\end{tabular}




\begin{tabular}{|c|c|c|c|c|c|c|}
\hline \multirow[b]{2}{*}{ Faixa etária } & \multicolumn{3}{|c|}{ FAV } & \multicolumn{3}{|c|}{ HOPE } \\
\hline & $\begin{array}{c}\text { Sem DMRI } \\
(\%)\end{array}$ & $\begin{array}{l}\text { DMRI inicial } \\
(\%)\end{array}$ & $\begin{array}{c}\text { DMRI estabelecida } \\
(\%)\end{array}$ & $\begin{array}{c}\text { Sem DMRI } \\
(\%)\end{array}$ & $\begin{array}{l}\text { DMRI inicial } \\
(\%)\end{array}$ & $\begin{array}{c}\text { DMRI estabelecida } \\
(\%)\end{array}$ \\
\hline 55-64 (anos) & 81 & 9 & 10 & 87 & 3 & 10 \\
\hline 65-74 (anos) & 69 & 19 & 12 & 65 & 23 & 12 \\
\hline$\geq 75$ (anos) & 40 & 15 & 45 & 73 & 3 & 24 \\
\hline
\end{tabular}

Foi observada associação significante entre DMRI e o gênero feminino $(\mathrm{p}=0,0451)$. Outrossim, não foi observada associação estatística entre DMRI e raça na amostra analisada $(\mathrm{p}=0,1367)$. A distribuição das freqüências de olhos com DMRI quanto ao gênero e raça, sem distinção da instituição estudada, está expressa na tabela 3.

A presença de doenças sistêmicas (hipertensão arterial sistêmica-HAS e diabetes melitus-DM) e associação com o tabagismo e ingestão alcoólica excessiva, na amostra estudada, estão expressos na tabela 4. Não houve associação estatística entre a presença de olhos com DMRI e HAS ( $\mathrm{p}=0,1985)$, $\mathrm{DM}(\mathrm{p}=0,6712)$, tabagismo $(\mathrm{p}=0,6374)$ e alcoolismo $(\mathrm{p}=0,7359)$ dos pacientes.

Foi estabelecida associação entre olhos com DMRI e obesidade $(\mathrm{p}<0,0001)$. Analisando-se a tabela 5 pode-se verificar baixa freqüência de DMRI estabelecida em indivíduos com IMC magro e normal, quando comparados a indivíduos com IMC anormal (acima do peso e obesos).

Em relação à exposição solar, foi observado que 176 olhos (88\%) dos pacientes da FAV tinham exposição ocupacional ao sol (agricultura), sendo este fato constatado em 16 olhos (8\%) dos pacientes do HOPE. A exposição solar esteve presente em 139 olhos sem DMRI (72\%), 22 olhos com DMRI inicial (12\%), 31 com DMRI Estabelecida (16\%), não havendo relação da exposição solar à presença de DMRI ou gravidade da doença $(\mathrm{p}=0,9399)$.

Não foi observada relação de DMRI e presença de íris clara $(\mathrm{p}=0,1691)$. Esse achado esteve presente em 57 olhos sem DMRI (84\%), 5 olhos com DMRI inicial (7\%), 6 com DMRI estabelecida $(9 \%)$.

\begin{tabular}{|c|c|c|c|c|c|c|c|c|c|c|}
\hline & \multicolumn{4}{|c|}{ Gênero } & \multicolumn{4}{|c|}{ Raça } & \multirow{2}{*}{\multicolumn{2}{|c|}{ Total }} \\
\hline & \multicolumn{2}{|c|}{ Masculino } & \multicolumn{2}{|c|}{ Feminino } & \multicolumn{2}{|c|}{ Leucodérmico } & \multicolumn{2}{|c|}{ Não-leucodérmico } & & \\
\hline & $\mathbf{n}$ & $\%$ & $\mathbf{n}$ & $\%$ & $\mathbf{n}$ & $\%$ & $n$ & $\%$ & $\mathbf{n}$ & $\%$ \\
\hline Sem DMRI & 94 & 32 & 199 & 68 & 163 & 56 & 130 & 44 & 293 & 100 \\
\hline Com DMRI & 46 & 43 & 61 & 57 & 69 & 64 & 38 & 36 & 107 & 100 \\
\hline
\end{tabular}

\begin{tabular}{|c|c|c|c|c|c|c|c|c|}
\hline & \multicolumn{2}{|c|}{ HAS } & \multicolumn{2}{|c|}{ DM } & \multicolumn{2}{|c|}{ Tabagismo } & \multicolumn{2}{|c|}{ Alcoolismo } \\
\hline & $\mathbf{n}$ & $\%$ & $\mathbf{n}$ & $\%$ & $\mathbf{n}$ & $\%$ & $\mathbf{n}$ & $\%$ \\
\hline Sem DMRI & 167 & 74 & 70 & 70 & 73 & 69 & 20 & 67 \\
\hline \multicolumn{9}{|l|}{ Com DMRI } \\
\hline Inicial & 15 & 7 & 10 & 10 & 12 & 11 & 4 & 13 \\
\hline Estabelecida & 44 & 19 & 20 & 20 & 21 & 20 & 6 & 20 \\
\hline Total & 226 & 100 & 100 & 100 & 106 & 100 & 30 & 100 \\
\hline
\end{tabular}

\begin{tabular}{|c|c|c|c|c|c|c|c|c|}
\hline & \multicolumn{2}{|c|}{ IMC magro } & \multicolumn{2}{|c|}{ IMC normal } & \multicolumn{2}{|c|}{ IMC acima peso } & \multicolumn{2}{|c|}{ IMC obeso } \\
\hline & $\mathbf{n}$ & $\%$ & $\mathbf{n}$ & $\%$ & $\mathbf{n}$ & $\%$ & $\mathbf{n}$ & $\%$ \\
\hline Sem DMRI & 15 & 62 & 104 & 67 & 130 & 83 & 44 & 67 \\
\hline \multicolumn{9}{|l|}{ Com DMRI } \\
\hline Inicial & 7 & 30 & 26 & 17 & 7 & 5 & 2 & 3 \\
\hline Estabelecida & 2 & 8 & 24 & 16 & 19 & 12 & 20 & 30 \\
\hline Total & 24 & 100 & 154 & 100 & 156 & 100 & 66 & 100 \\
\hline
\end{tabular}


As freqüências de olhos fácicos e pseudofácicos e presença de catarata e sua relação com a presença de DMRI e o estágio da doença estão expressas na tabela 6 . Houve associação estatística entre DMRI e catarata $(\mathrm{p}=0,0447)$ e pseudofacia $(\mathrm{p}=0,0432)$.

$\mathrm{O}$ uso regular de vitaminas foi observada em $2 \%$ da população da FAV e em 14\% da população do HOPE. Nos pacientes usuários de vitaminas, foi constatado que $75 \%$ deles não possuíam DMRI, $13 \%$ tinham DMRI inicial e 12\% DMRI estabelecida. Não foi observada associação estatística entre o uso de vitaminas e baixa freqüência de DMRI na amostra estudada $(\mathrm{p}=0,8251)$.

Em relação à distribuição dos erros refrativos da população estudada foi observada presença de hipermetropia em 53\% dos olhos sem DMRI, em 69\% com DMRI inicial e em 66\% com DMRI estabelecida. A miopia esteve presente em $4 \%$ dos olhos sem DMRI, em 21\% com DMRI inicial e em $6 \%$ com DMRI estabelecida. Foi observada que na presença de DMRI houve maior ocorrência de hipermetropia. Entretanto esse achado não foi significante, não se podendo estabelecer uma relação estatística entre DMRI e hipermetropia $(\mathrm{p}=0,5098)$.

\section{DISCUSSÃO}

A DMRI constitui uma importante causa de cegueira em pacientes acima de 55 anos. No presente estudo foi observada prevalência distinta entre as duas populações socioeconômicas estudadas, com maior freqüência de DMRI no setor público, sem, entretanto, relação estatística da doença com o nível socioeconômico da população. Não se tem descrito na literatura associação entre DMRI e nível socioeconômico, assim como estudos não têm evidenciado associação entre DMRI e nível de escolaridade ${ }^{(4)}$.

Avaliando-se a freqüência de DMRI nas diversas faixas etárias, na presente análise, pode-se observar que os resultados obtidos diferem da literatura que apontam frequiências menores nas suas casuísticas ${ }^{(1,12,15)}$. A limitação da amostragem do presente estudo e a diversidade dos critérios na literatura, para definição de DMRI, deve ser levada em consideração na análise crítica dos dados.

Dentre os vários fatores de risco para DMRI, a idade é um fator determinante da doença $a^{(1,4)}$. Os resultados da presente análise ratificam os resultados da literatura. Em relação à associação de DMRI e gênero, foi observado no presente estudo, associação da doença com o gênero feminino, o que é confirmado por alguns autores ${ }^{(4)}$. Não foi encontrada associação de DMRI e raça no presente estudo.

Em relação aos fatores de risco, a presença de comorbidades sistêmicas como hipertensão arterial sistêmica e diabetes mellitus não foi associada a DMRI na presente casuística em semelhança aos achados de outros autores ${ }^{(4)}$. Ademais, não se pode estabelecer relação de DMRI e presença de tabagismo, diferindo dos achados da literatura ${ }^{(4-5)}$ e não se pode estabelecer relação de DMRI e alcoolismo, o que ratifica os achados de McArty ${ }^{4}$ e vai de encontro aos resultados de estudos específi$\cos ^{(8)}$. A associação entre DMRI e exposição solar relatada em estudos da literatura e refutada em outros ${ }^{(4)}$, não foi observada na presente análise. A associação entre DMRI e presença de catarata ou cirurgia de catarata estabelecida na presente análise é um fato controverso na literatura ${ }^{(4)}$. Apesar de ter sido descrito na literatura que dietas balanceadas, ricas em antioxidantes ${ }^{(9-11,16)}$ podem produzir um efeito benéfico no curso progressivo da DMRI, não foi obtida relação entre o uso de vitaminas e baixa frequiência de DMRI na amostra estudada. Por fim, a associação entre íris clara e DMRI, não demonstrada consistentemente por estudos da literatura ${ }^{(4)}$, também não foi confirmada no presente estudo. Tem sido sugerido que, embora não haja correlação direta entre íris clara e DMRI, pode haver relação indireta devido à sensibilidade da pele desses indivíduos ao sol e habilidade dos tecidos em reparar danos induzidos pelo mesmo ${ }^{(4)}$.

Outro fator de risco fortemente relacionado a presença de DMRI e progressão para formas avançadas da doença constituiu IMC acima do peso (25-29) e IMC obeso ( $\geq 30)$. Este achado ratifica dados da literatura nos quais o aumento do índice de massa corpóreo (IMC) e aumento da circunferência abdominal estão associados a aumentado risco de progressão para formas avançadas de DMRI, enquanto que a atividade física está associada à reduzida taxa de progressão( ${ }^{(7)}$.

Por fim é bom salientar as limitações de se estabelecer uma relação entre DMRI e os fatores de risco associados, devido ao tamanho da amostra da presente análise.

\section{CONCLUSÕES}

A frequiência de DMRI nos dois centros oftalmológicos estudados variou de 23 a $30 \%$, observando-se associação desta doença com a idade, gênero feminino, catarata, cirurgia de catarata e obesidade, o qual é o único fator de risco modifi-

\begin{tabular}{|c|c|c|c|c|c|c|}
\hline & \multicolumn{2}{|c|}{ Fácico } & \multicolumn{2}{|c|}{ Pseudofácico } & \multicolumn{2}{|c|}{ Catarata } \\
\hline & $\mathbf{n}$ & $\%$ & $\mathbf{n}$ & $\%$ & $\mathbf{n}$ & $\%$ \\
\hline Sem DMRI & 231 & 70 & 59 & 86 & 77 & 62 \\
\hline \multicolumn{7}{|l|}{ Com DMRI } \\
\hline Inicial & 40 & 12 & 2 & 3 & 19 & 15 \\
\hline Estabelecida & 58 & 18 & 7 & 11 & 29 & 23 \\
\hline Total & 329 & 100 & 68 & 100 & 125 & 100 \\
\hline
\end{tabular}


cável. Não se obteve relação entre essa doença e nível socioeconômico, raça, hipertensão arterial sistêmica, diabetes mellitus, tabagismo, alcoolismo, hipermetropia, exposição solar, cor clara de íris e uso crônico de vitaminas antioxidantes.

\section{ABSTRACT}

Purpose: To evaluate age-related macular degeneration (AMD) prevalence and its risk factors in two different populations, with distinct socioeconomic conditions, at two ophthalmological centers in Pernambuco state, Brazil. Methods: A cross-sectional study was performed in 200 volunteers aged 55 years or older. One hundred were cared for by the Public Health System (in the Fundação Altino Ventura FAV-SUS) and 100 were cared for at a private hospital (Hospital de Olhos de Pernambuco - HOPE). There were 400 eyes altogether. Age-related macular degeneration was graded using fundoscopic examination using AREDS classification (Age-Related Eye Disease Study). Results: Presence of age-related macular degeneration was observed in 61 eyes $(30 \%)$ of the patients of the Fundação Altino Ventura and in 46 eyes (23\%) of the patients of the Hospital de Olhos de Pernambuco. The age-related macular degeneration frequency difference between these populations, that are not similar regarding their socioeconomic level, was not significant $(\mathrm{p}=0.113)$. There was a positive association between aging and the agerelated macular degeneration frequency $(\mathrm{p}<0.0001)$. This association was also present in the female gender $(\mathrm{p}=0.0451)$, the presence of cataract $(\mathrm{p}=0.0447)$, cataract surgery $(\mathrm{p}=0.0432)$ and obesity ( $\mathrm{p}<0.0001)$. No age-related macular degeneration association with race $(\mathrm{p}=0.1367)$, hypertension $(\mathrm{p}=0.1985)$, diabetes ( $\mathrm{p}=0.6712)$, smoking $(\mathrm{p}=0.6374)$, alcohol intake $(\mathrm{p}=0.7359)$, ocular sunlight exposure $(\mathrm{p}=0.9399)$, light-colored iris $(\mathrm{p}=0.1691)$, hyperopia ( $\mathrm{p}=0.5098)$ and the usage of antioxidants $(\mathrm{p}=0.8251)$ was observed. Conclusions: The age-related macular degeneration frequency in the two studied ophthalmological centers varied from 23 to $30 \%$. Age-related macular degeneration association with age; female gender; cataract; cataract surgery; and obesity was observed. No age-related macular degeneration association with socioeconomic level was found in this study.
Keywords: Macular degeneration/epidemiology; Risk factors; Aging; Aged; Obesity; Alcohol intake; Smoking; Socioeconomic factors

\section{REFERÊNCIAS}

1. Soubrane G, Haddad WM, Coscas G. Age-related macular degeneration. Presse Med. 2002;31(27):1282-7. Francese

2. Evans $\mathrm{J}$, Wormald $\mathrm{R}$. Is the incidence of registrable age-related macular degeneration increasing? Br J Ophthalmol. 1996;80(1):9-14. Comment in: $\mathrm{Br}$ J Ophthalmol. 1996;80(1):2-3.

3. Bone RA, Landrum JT, Guerra LH, Ruiz CA. Lutein and zeaxanthin dietary supplements raise macular pigment density and serum concentrations of these carotenoids in humans. J Nutr. 2003;133(4):992-8. Erratum in: J Nutr. 2003; 133(6): 1953.

4. McArty CA, Mukesh BN, Fu CL, Mitchell P, Wang JJ, Taylor HR. Risk factors for age-related maculopathy: the visual impairment project. Arch Ophthalmol. 2001;119(10):1455-62.

5. Delcourt C, Cristol JP, Tessier F, Léger CL, Descomps B, Papoz L, et al. Agerelated macular degeneration and antioxidant status in the POLA study. Arch Ophthalmol. 1999;117(10):1384-90.

6. Hyman L, Schachat AP, He Q, Leske MC. Hypertension, cardiovascular disease, and age-related macular degeneration. Arch Ophthalmol. 2000;118(3):351-8.

7. Seddon JM, Cote J, Davis N, Rosner B. Progression of age-related macular degeneration: association with body mass index, waist circumference, and waist-hip ratio. Arch Ophthalmol. 2003;121(6):785-92.

8. Cho E, Hankinson SE, Willet WC, Stampfer MJ, Spiegelman D, Speizer FE, et al. Prospective study of alcohol consumption and the risk of age-related macular degeneration. Arch Ophthalmol. 2000;118(5):681-8.

9. Pratt S. Dietary prevention of age-related macular degeneration. J Am Optom Assoc. 1999;70(1):39-47.

10. A randomized, placebo-controlled, clinical trial of high-dose supplementation with vitamins $\mathrm{C}$ and $\mathrm{E}$, beta carotene, and zinc for age-related macular degeneration and vision loss. AREDS Report 8. Arch Ophthalmol. 2001;119(10): 1417-36. Comment in: Arch Ophthalmol. 2001;119(10):1533-4.

11. Van der Hagen AM, Yolton DP, Kaminski MS, Yolton RL. Free radicals and antioxidant supplementation: a review of their roles in age-related macular degeneration. J Am Optom Assoc. 1993;64(12):871-8.

12. Oneill C, Jamison J, McCulloch D, Smith D. Age-related macular degeneration: cost-of-illness issues. Drugs Aging. 2001;18(4):233-41.

13. Koh AH, Ang CL. Age-related macular degeneration: what's new. Ann Acad Med Singapore. 2002;31(3):399-404.

14. Milton JS, Tsokos JO. Statistical methods in the biological and health sciences. In: Milton JS, Tsokos JO. Categorical data. New York: Mcgraw-Hill International Book; 1983. p.381-411.

15. Margherio RR, Margherio AR, DeSantis ME. Laser treatments with verteporfin therapy and its potential impact on retinal practices. Retina. 2000;20(4):325-30.

16. Richer S. Multicenter ophthalmic and nutritional age-related macular degeneration study - part 2: antioxidant intervention and conclusions. J Am Optom Assoc. 1996;67(1):30-49.

\section{ABO ELETRÔNICO}

\section{Acesso: http://www.abonet.com.br}

